\title{
Propiedades termodinámicas de sorción de agua de la pulpa de lulo en polvo con encapsulantes
}

\author{
Propriedades termodinâmicas de sorção de água de polpa de lulo em pó com encapsulantes
}

Ana Lúcia GABAS*, Javier TELIS-ROMERO², Gloria Ines GIRALDO-GÓMEZ1 , Vânia Regina Nicoletti TELIS²

\section{Resumen}

Datos de humedad de equilibrio de polvo de pulpa de lulo (PL) con y sin aditivos - 58\% de maltodextrina (MD) o 58\% de goma Arábiga (GA) - fueran determinados en las temperaturas de $20,30,40$ y $50{ }^{\circ} \mathrm{C}$ utilizando el método estático gravimétrico para un rango de actividades de agua entre 0.06 y 0.90. Las isotermas presentaran formato sigmoidal del tipo III, y el modelo de Guggenhein-Anderson-de Boer (GAB) fue ajustado satisfactoriamente a los datos experimentales de humedad de equilibrio en función de la actividad de agua. La adición de encapsulantes afecto las isotermas de tal manera que en la misma actividad de agua, las muestras PL + GA y PL + MD presentaron un menor contenido de humedad de equilibrio y no fueron afectadas por la variación de temperatura. Los calores isostéricos de sorción de las pulpas en polvo con encapsulantes fueron mayores (menos negativos) con relación a las pulpas de lulo en polvo, sugiriendo la existencia de sitios polares más activos en el producto sin adición de GA o MD. Una relación exponencial empírica fue utilizada para describir la dependencia del calor de sorción con el contenido de humedad del material.

Palabras-clave: actividad de agua; calor de sorción; goma Arábiga; isoterma; lulo; maltodextrina.

\section{Resumo}

Dados de umidade de equilíbrio de polpa de lulo em pó (PL) com e sem aditivos - 58\% de maltodextrina (MD) ou 58\% de goma Arábica (GA) - foram determinados nas temperaturas de $20,30,40$ e $50^{\circ} \mathrm{C}$, utilizando-se o método estático gravimétrico numa faixa de atividade de água de 0,06 a 0,90. As isotermas apresentaram formato sigmoidal do tipo III e o modelo de Guggenhein-Anderson-de Boer (GAB) ajustou satisfatoriamente os dados experimentais de umidade de equilíbrio em função da atividade de água. A adição de encapsulantes afetou as isotermas de tal maneira que na mesma atividade de água, as amostras PL + GA y PL + MD apresentaram um menor conteúdo de umidade de equilíbrio e não foram afetadas pela variação de temperatura. Os calores isostéricos de sorção das polpas em pó com encapsulantes foram maiores (menos negativos) em relação à polpa de lulo em pó, sugerindo a existência de sítios polares mais ativos no produto sem adição de GA ou MD. Uma relação exponencial empírica foi utilizada para descrever a dependência do calor de sorção com o conteúdo de umidade do material.

Palavras-chave: atividade de água; calor de sorção; goma Arábica; isoterma; lulo; maltodextrina.

\section{Introducción}

El lulo de castilla o naranjilla (Solanum quitoense Lam) es una planta originaria de los Andes Sudamericanos, típica en Colombia en las regiones húmedas de clima medio y frio moderado, en donde crece en forma expontánea o en siembra. Su cultivo aunque muy incipiente se encuentra a lo largo del callejón interandino entre Colombia y Ecuador, este se desarrolla bien entre los 1600 y $2500 \mathrm{~m}$ de altitud con temperaturas entre los 22 y $25^{\circ} \mathrm{C}$. De acuerdo con los estudios de la Comunidad Económica Europea, el lulo es una de las frutas andinas con mayor posibilidad de exportación (TAMAYO et al., 2001).

El lulo presenta una variación de tamaño de peso entre 18 y 191 g, diámetro ecuatorial entre 32 y 78 mm e de diámetro polar entre 29 y $72 \mathrm{~mm}$, el contenido de pulpa de la fruta está entre el 68 y el 72\%. Las características fisico-químicas de la pulpa son: $\mathrm{pH}$ alrededor de 2.5 , ${ }^{\circ}$ Brix entre 7 y 10 dependiendo del grado de madurez de la fruta, acidez titulable expresada como porcentaje de ácido cítrico del 3\% (GÓMEZ et al., 2004).

Los polvos producidos a partir de las pulpas de frutas deshidratadas son productos estables, fáciles de manipular, se reconstituyen rápidamente formando productos con propiedades similares a las de los jugos originales. Estos productos son usados principalmente como complementos alimenticios y tienen larga vida de almacenamiento a temperaturas normales.

El secado de jugo de frutas y otros productos con altos contenidos de azúcar presenta dificultades técnicas debido a su elevada higroscopicidad y termo-plasticidad en altas temperaturas y humedades (ADHIKARI et al., 2004). Por esta razón, la adición de maltodextrina, gomas y otras substancias como pectinas, silicato de calcio, y carboxy-methyl celulosa,

Recebido para publicação em 12/3/2008

Aceito para publicação em 3/1/2009 (003304)

${ }^{1}$ Departamento de Física e Química, Universidad Nacional de Colombia, Manizales - Colombia

2 Departamento de Engenharia e Tecnologia de Alimentos, Universidade Estadual Paulista - UNESP, São José do Rio Preto - SP, Brasil

${ }_{3}^{3}$ Engenharia de Alimentos, Universidade de São Paulo - USP, Pirassununga - SP, E-mail: gabas@usp.br

${ }^{*}$ A quem a correspondência deve ser enviada 
han sido utilizadas en la producción de jugos en polvo (BHANDARI et al., 1993).

Las maltodextrinas son polímeros de glucosa obtenidos a partir del almidón mediante procesos enzimáticos. Son unidades de $\beta$-D-glucosa unidas principalmente por enlaces glucosídicos $(1 \rightarrow 4)$ y generalmente están clasificadas de acuerdo con su equivalente de dextrosa (DE), el cual determina su capacidad de reducción que está inversamente relacionado con su peso molecular medio (BEMILLER; WHISTLER, 1996). Las maltodextrinas son utilizadas principalmente en materiales que presentan dificultades para su deshidratación, tales como jugo de frutas, condimentos y endulzantes (REINECCIUS, 1991), ya que reducen los problemas de adherencia y aglomeración durante el almacenamiento, mejorando así la estabilidad del producto (BHANDARI et al., 1993; SILVA; SOBRAL; KIECKBUSH, 2006).

La goma arábiga es un complejo heteropolisacárido con una estructura altamente ramificada; su cadena principal está formada por unidades de D-galactopiranosa unidas mediante enlaces $\beta$-D glucosídicos $(1 \rightarrow 3)$. Las cadenas laterales con estructuras químicas variables, formadas a partir de unidades de D-galactopiranosa, L-ranosa, L-arabinofuranosa y ácido $\mathrm{D}$-galacturónico, están unidas a la cadena principal por enlaces $\beta$ - $(1 \rightarrow 6)$ (BEMILLER; WHISTLER, 1996). La goma arábiga ha sido utilizada como material encapsulante durante el secado por atomización, debido a su buena capacidad emulsificante y baja viscosidad en soluciones acuosas. Además, proporciona una buena retención de substancias volátiles y confiere una efectiva protección contra la oxidación. Aunque la goma arábiga está siendo utilizada como agente encapsulante, su contribución en la estabilidad de alimentos deshidratados o encapsulados no ha sido muy estudiada (RIGHETTO; NETTO, 2005).

La actividad de agua y el contenido de humedad del alimento se relacionan a través de la isoterma de sorción, que es la curva que indica en el equilibrio y para una temperatura dada, la cantidad de agua retenida por un alimento en función de la humedad relativa de la atmósfera que lo rodea (ROCKLAND, 1987).

El conocimiento de las isotermas de sorción de agua y el calor isostérico de sorción son esencialmente importantes para varios procesos de alimentos, como secado, almacenamiento y embalajes, ya que ayudan a calcular el tiempo de secado, predecir el comportamiento de los componentes en una mezcla hecha para producir un alimento procesado, a partir de las curvas de los componentes puros; además seleccionar el material de empaque adecuado, modelar la variación de humedad durante el almacenamiento y estimar la vida de anaquel en productos de baja humedad (LOMAURO; BAKSHI; LABUZA, 1985).

Estas propiedades dan información acerca de los mecanismos de sorción y las interacciones entre los componentes del alimento y el agua. También ayudan a establecer el contenido de humedad final de un producto y permiten calcular la energía requerida en la operación unitaria de secado (GABAS; MENEGALLI; TELIS-ROMERO, 2000).

En la literatura se encuentran numerosos modelos matemáticos para describir el comportamiento de sorción del agua en los alimentos. Algunos son presentados en el trabajo de Lopes-Filho et al. (2002). Varios de estos modelos están basados en teorías de mecanismos de sorción; otros son puramente empíricos o semi-empíricos. El criterio usado para seleccionar el modelo más apropiado es el grado de ajuste a los datos experimentales y su significado físico.

El objetivo de este trabajo fue determinar experimentalmente las isotermas de sorción de vapor de agua en deshidratados de jugo de lulo obtenidos por secado a vacío en un rango de temperaturas de 20 a $50{ }^{\circ} \mathrm{C}$, y analizar el efecto de la adición de maltodextrina y goma arábiga sobre las propiedades termodinámicas de la sorción de vapor de agua.

\subsection{Desarrollo matemático}

Para ajustar los datos experimentales de las isotermas de sorción de vapor de agua de cada uno de los productos deshidratados, se utilizó la ecuación de GAB (GuggenheimAnderson-de Boer). Este modelo se expresa por la Ecuación 1.

$\mathrm{X}=\frac{(\mathrm{C}-1) \cdot \mathrm{K} \cdot \mathrm{a}_{\mathrm{w}} \cdot \mathrm{X}_{\mathrm{m}}}{1+(\mathrm{C}-1) \cdot \mathrm{K} \cdot \mathrm{a}_{\mathrm{w}}}+\frac{\mathrm{K} \cdot \mathrm{a}_{\mathrm{w}} \cdot \mathrm{X}_{\mathrm{m}}}{1-\mathrm{K} \cdot \mathrm{a}_{\mathrm{w}}}$

donde $\mathrm{a}_{\mathrm{w}}$ es la actividad de agua; $\mathrm{X}_{\mathrm{m}}$ es el contenido de humedad (base seca) correspondiente a la monocapa (BET) y C y K son constantes relacionadas con el efecto de la temperatura (Ecuación 2 y 3 ).

$\mathrm{C}=\mathrm{C}_{\mathrm{G}} \exp \left(\frac{\Delta \mathrm{H}_{\mathrm{c}}}{\mathrm{RT}}\right)$

$\mathrm{K}=\mathrm{K}_{\mathrm{G}} \exp \left(\frac{\Delta \mathrm{H}_{\mathrm{k}}}{\mathrm{RT}}\right)$

donde $\Delta \mathrm{H}_{\mathrm{c}}$ y $\Delta \mathrm{H}_{\mathrm{k}}$ son funciones del calor de sorción de agua: $\Delta \mathrm{H}_{\mathrm{c}}=\mathrm{H}_{\mathrm{m}}-\mathrm{H}_{\mathrm{n}}$ y $\Delta \mathrm{H}_{\mathrm{k}}=\lambda-\mathrm{H}_{\mathrm{n}}$. En las ecuaciones anteriores $\mathrm{C}_{\mathrm{G}}$ $\mathrm{y} \mathrm{K}_{\mathrm{G}}$ son constantes ajustadas en función de la temperatura, $\mathrm{H}_{\mathrm{m}}$ $\mathrm{y}_{\mathrm{n}}$ son respectivamente el calor de sorción de la monocapa y multicapa, y $\lambda$ el calor latente de condensación del agua pura.

Para estimar los parámetros, los datos experimentales se ajustaron al modelo matemático usando regresión no lineal en el software Statistica (STATSOFT, 1995). El ajuste de la función se evaluó con el coeficiente de correlación $\left(\mathrm{R}^{2}\right)$ y la suma del cuadrado de los residuos (SSR).

\subsection{Propiedades termodinámicas}

El calor isostérico de sorción o entalpía de sorción $\left(\mathrm{q}_{\mathrm{st}}\right)$ se define como la diferencia entre el calor total de sorción $\left(Q_{\mathrm{st}}\right)$ y el calor latente de vaporización del agua. Se puede determinar mediante la Ecuación 4, derivada de la ecuación de ClausiusClapeyron (RIZVI, 1995):

$\left.\frac{\partial \ln \left(\mathrm{a}_{\mathrm{w}}\right)}{\partial(1 / \mathrm{T})}\right|_{\mathrm{X}}=\frac{\mathrm{Q}_{\mathrm{st}}-\lambda}{\mathrm{R}}=\frac{\mathrm{q}_{\mathrm{st}}}{\mathrm{R}}$ 
donde $\mathrm{a}_{\mathrm{w}}$ es la actividad de agua; $\mathrm{T}$ es la temperatura absoluta; $\mathrm{R}$ es la constante universal de los gases, $\mathrm{Q}_{\mathrm{st}}$, el calor integral de desorción del sólido húmedo y es el calor latente de vaporización del agua pura.

El calor isostérico de sorción es una cantidad diferencial molar derivada de la dependencia que presentan las isotermas de sorción con respecto a la temperatura. Para su aplicación se requiere la medida de las isotermas a 2 o más temperaturas (MCLAUGHLIN; MAGEE, 1998). Tsami et al. (1990), presentan una relación exponencial empírica entre el calor isostérico de sorción y el contenido de humedad. Esta se muestra en la Ecuación 5.

$\mathrm{q}_{\mathrm{st}}=\mathrm{q}_{0} \exp \left(-\mathrm{X}_{\mathrm{eq}} / \mathrm{X}_{0}\right)$

donde $\mathrm{q}_{0}$ es el calor isostérico de sorción de la monocapa molecular de agua, $\mathrm{X}_{\mathrm{eq}}$ es el contenido de humedad de equilibrio, $\mathrm{X}_{0}$ es el contenido de humedad inicial del material alimenticio.

\section{Material y métodos}

La fruta a partir de la cual se obtuvo la pulpa presentó las siguientes características: $(8.6 \pm 0.4){ }^{\circ}$ Brix, $(67.6 \pm 1.3) \mathrm{g} .100 \mathrm{~g}^{-1}$ contenido de pulpa, $\mathrm{pH} 2.42 \pm 0.03,(2.98 \pm 0.02) \mathrm{g} .100 \mathrm{~g}^{-1}$ ácido cítrico y densidad $(1.0488 \pm 0.0038)$ g.cm $\mathrm{cm}^{-3}$. Para la obtención de la pulpa se utilizó una despulpadora piloto y fue tamizada a través de una malla de $1.6 \mathrm{~mm}$.

Se preparó una solución acuosa de maltodextrina comercial MOR-REX 1920 (Corn Products Brazil) a una concentración del $70 \%$ en masa en agua destilada a $40{ }^{\circ} \mathrm{C}$, usando un agitador mecánico. Esta solución fue adicionada a la pulpa de lulo de tal forma que la relación másica entre la solución de maltodextrina y pulpa fuera del $58 \%$ en base seca (58 g de maltodextrin/100 g de sólidos totales). Según las especificaciones técnicas del fabricante, la maltodextrina MOR-REX ${ }^{\oplus} 1920$ presenta $17.0 \leq \mathrm{DE} \leq 19.9$.

Se usó un procedimiento similar para preparar la pulpa de lulo con 55\% de contenido de goma arábiga (GA) (Synth, Brazil) en base seca (55 g de goma Arábiga/100 g de sólidos totales). Pulpas de Lulo (PL) con y sin aditivos fueron deshidratadas en bandejas de acero inoxidable, en una cámara de vacío (26 in Hg, $60{ }^{\circ} \mathrm{C}$ ) durante 48 horas. El producto deshidratado fue molido en un mortero de porcelana.

Para obtener las isotermas de sorción se empleó el método gravimétrico estático, para ello se determinó el contenido de humedad de equilibrio de la pulpa deshidratada a diferentes actividades de agua (JOWITT et al., 1983), a temperaturas de 20, 30,40 y $50{ }^{\circ} \mathrm{C}$. Se prepararon diez soluciones saturadas de sales que cubren un rango de actividad de agua entre 0.06 y 0.90. Cada solución saturada fue transferida a desecadores individuales de vidrio, de forma que la cantidad de solución alcanzara una altura de aproximadamente $1.5 \mathrm{~cm}$. Se pesaron por triplicado muestras de cerca de $1 \mathrm{~g}$ de polvo en pequeños recipientes de plástico, se colocaron sobre un trípode en los desecadores de vidrio los cuales se cerraron herméticamente y se colocaron en una cámara a temperatura controlada. Las muestras fueron pesadas periódicamente hasta alcanzar el equilibrio, que se considera cuando la diferencia en peso entre dos pesadas consecutivas no excede el $0.1 \%$. El tiempo requerido para alcanzar el equilibrio fue de 4 a 5 semanas. La humedad de equilibrio se determinó en una estufa a vacío a temperatura de $60{ }^{\circ} \mathrm{C}$ por 48 horas (AOAC, 1990).

\section{Resultados y discusiones}

Los contenidos de humedad de equilibrio en función de la actividad de agua para polvos de lulo con y sin aditivos a diferentes temperaturas se presentan en la Tabla 1. El valor representa un promedio de tres 3 repeticiones, con una error máximo del 5.4\%.

Para ajustar los datos experimentales de humedad de equilibrio en función de la actividad de agua, se seleccionó el modelo de GAB (Ecuación 1), ampliamente utilizado para alimentos principalmente para frutas (GABAS; TELIS-ROMERO; MENEGALLI, 1999; TELIS et al., 2000). La Figura 1 presenta las isotermas de sorción experimentales para polvo de lulo a cada temperatura con sus respectivos ajustes al modelo de GAB. Como se puede observar, el comportamiento de todas las curvas fue del tipo III, de acuerdo con la clasificación de Brunauer (RIZVI, 1995). Los resultados de la regresión no-lineal para el ajuste del modelo de GAB a los datos experimentales son presentados en las Tablas 2, 3 y 4.

Los resultados obtenidos en este trabajo con relación al ajuste del modelo de GAB utilizando diferentes temperaturas están de acuerdo con los valores límites de las constantes $\mathrm{C}$ y K sugeridos por Lewicki (1997), basado en el análisis matemático del modelo. Como forma de garantizar una buena descripción de las isotermas del tipo sigmoidal y alcanzar los requerimientos del modelo de BET, así como asegurar que el cálculo de humedad de la monocapa $\left(\mathrm{X}_{\mathrm{m}}\right)$ no difiera en $\pm 15.5 \%$ del valor verdadero, este autor establece que las constantes deben asumir valores en un rango entre $0.24 \leq \mathrm{K} \leq 1$ y $5.67 \leq \mathrm{C} \leq \infty$.

El valor verdadero de la humedad de la monocapa $\left(\mathrm{X}_{\mathrm{m}}\right)$ es de interés particular, ya que este indica la cantidad de agua que está fuertemente adsorbida en sitios específicos de la superficie del alimento y es considerado como el valor óptimo para asegurar su estabilidad. Para lulo puro, los resultados presentan valores de $\mathrm{X}_{\mathrm{m}}$ en el rango entre 12.7 - 15.4\% (base seca) con una tendencia a disminuir con el aumento de la temperatura de 20 a $50{ }^{\circ} \mathrm{C}$. Nicoleti, Telis-Romero y Telis (2001) encontraron valores de $\mathrm{X}_{\mathrm{m}}$ para piña en trozos entre 6.3 y $13.2 \%$ (base seca), con experimentos de desorción a temperaturas en el rango de $40{ }^{\circ} \mathrm{C}$ a $70{ }^{\circ} \mathrm{C}$, por su parte Telis y Sobral (2001) obtuvieron valores de $\mathrm{X}_{\mathrm{m}}=7.2 \%$ (base seca) en la desorción a $25^{\circ} \mathrm{C}$, para el producto deshidratado por liofilización. Las diferencias entre los valores de $\mathrm{X}_{\mathrm{m}}$ obtenidos para frutas en diferentes trabajos pueden ser atribuidas a variación en la composición de la materia prima, principalmente el contenido de azúcares. Para algunas otras frutas, como uvas (GABAS; TELIS-ROMERO; MENEGALLI, 1999), caqui (TELIS et al., 2000), ciruelas (GABAS; MENEGALLI; TELIS-ROMERO, 2000), membrillo y níspero (MOREIRA et al., 2008) se encontraron valores en el 
Tabla 1. Humedad de equilibrio experimental $\left(\mathrm{kg} \mathrm{H}_{2} \mathrm{O}_{\mathrm{kg}}{ }^{-1}\right.$ materia seca) para polvos de pulpa de lulo.

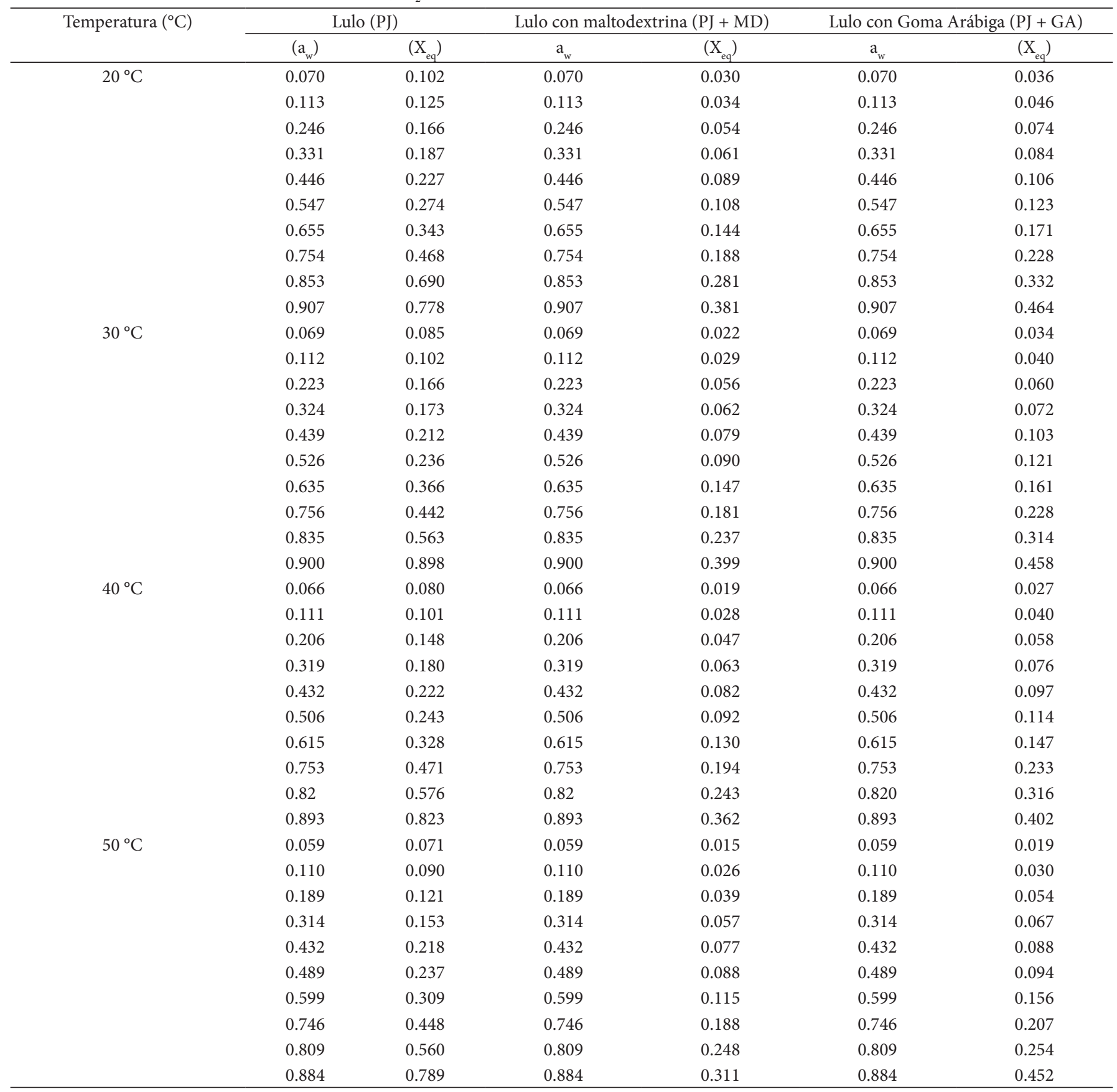

rango entre 8.0 y 16.3 (\% base seca), lo cual está en concordancia con el orden de magnitud de $\mathrm{X}_{\mathrm{m}}$ calculado en este trabajo.

Las fuertes interacciones adsorbente-adsorbato, las cuales son exotérmicas, son favorecidas en temperaturas mas bajas, causando un aumento en el parámetro C con la disminución de la temperatura (DIOSADY et al., 1996), ajustándose con la Ecuación 4 que describe la dependencia de $\mathrm{C}$ con la temperatura. Resultados semejantes fueron encontrados en este trabajo: un aumento en la temperatura de 20 a $50{ }^{\circ} \mathrm{C}$ produce una disminución en $\mathrm{C}$ para todas las muestras.
El valor de K proporciona una medida de las interacciones entre las moléculas del agua de la multicapa con el adsorbente, y su valor tiende a estar entre el de la energía de las moléculas en la monocapa y el del agua líquida. Si K es igual a 1, las multicapas tienen propiedades de agua líquida (PÉREZ-ALONSO et al., 2006) y el comportamiento de sorción puede ser modelado por la ecuación de BET (SOBRAL; LEBERT; BIMBENET, 1999). Los valores de K de polvo de pulpa de lulo puro presentaron pequeñas variaciones en el rango de 0.894 a 0.951 . 
Generalmente hay una tendencia del contenido de humedad de equilibrio a disminuir con el aumento de la temperatura en actividades de agua constantes. La magnitud de la disminución

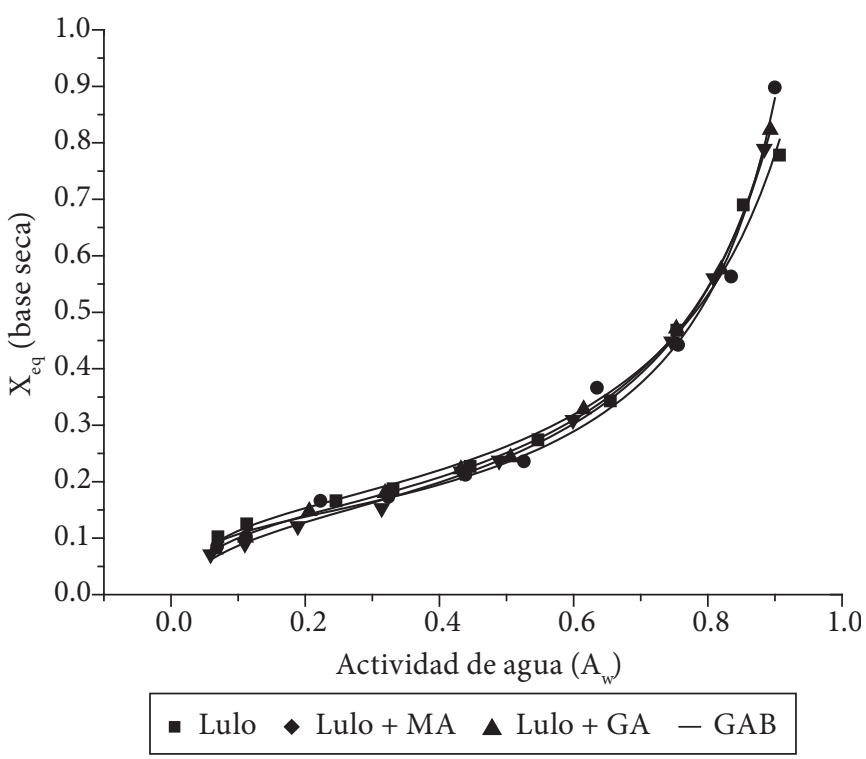

Figura 1. Isotermas de pulpa de lulo en diferentes temperaturas.

Tabla 2. Parámetros estimados del modelo de GAB para polvo de pulpa de lulo en diferentes temperaturas.

\begin{tabular}{ccccc}
\hline Parámetro & \multicolumn{4}{c}{ Temperatura } \\
\cline { 2 - 5 } & $20{ }^{\circ} \mathrm{C}$ & $30{ }^{\circ} \mathrm{C}$ & $40{ }^{\circ} \mathrm{C}$ & $50{ }^{\circ} \mathrm{C}$ \\
\hline $\mathrm{C}$ & 20.35 & 30.60 & 16.63 & 11.94 \\
$\mathrm{~K}$ & 0.894 & 0.951 & 0.925 & 0.930 \\
$\mathrm{X}_{\mathrm{m}}$ & 0.154 & 0.127 & 0.144 & 0.142 \\
$\mathrm{SSR}$ & $4.10 \times 10^{-3}$ & $6.31 \times 10^{-3}$ & $4.18 \times 10^{-4}$ & $3.95 \times 10^{-4}$ \\
$\mathrm{R}^{2}$ & 0.991 & 0.989 & 0.999 & 0.999 \\
\hline
\end{tabular}

Tabla 3. Parámetros estimados del modelo de GAB para polvo de pulpa de lulo con $58 \%$ de maltodextrina en diferentes temperaturas.

\begin{tabular}{ccccc}
\hline Parámetro & \multicolumn{4}{c}{ Temperatura } \\
\cline { 2 - 5 } & $20{ }^{\circ} \mathrm{C}$ & $30{ }^{\circ} \mathrm{C}$ & $40{ }^{\circ} \mathrm{C}$ & $50{ }^{\circ} \mathrm{C}$ \\
\hline $\mathrm{C}$ & 9.81 & 13.55 & 6.85 & 3.11 \\
$\mathrm{~K}$ & 0.939 & 0.973 & 0.942 & 0.886 \\
$\mathrm{X}_{\mathrm{m}}$ & 0.057 & 0.049 & 0.058 & 0.074 \\
$\mathrm{SSR}$ & $9.69 \times 10^{-5}$ & $1.17 \times 10^{-3}$ & $7.52 \times 10^{-5}$ & $3.95 \times 10^{-4}$ \\
$\mathrm{R}^{2}$ & 0.999 & 0.990 & 0.999 & 0.995 \\
\hline
\end{tabular}

Tabla 4. Parámetros estimados del modelo de GAB para polvo de pulpa de lulo con $58 \%$ de goma arábiga en diferentes temperaturas.

\begin{tabular}{ccccc}
\hline Parámetro & \multicolumn{4}{c}{ Temperatura } \\
\cline { 2 - 5 } & $20{ }^{\circ} \mathrm{C}$ & $30{ }^{\circ} \mathrm{C}$ & $40{ }^{\circ} \mathrm{C}$ & $50{ }^{\circ} \mathrm{C}$ \\
\hline $\mathrm{C}$ & 15.15 & 10.26 & 5.54 & 11.10 \\
$\mathrm{~K}$ & 0.946 & 0.953 & 0.912 & 0.992 \\
$\mathrm{X}_{\mathrm{m}}$ & 0.065 & 0.066 & 0.078 & 0.055 \\
$\mathrm{SSR}$ & $1.142 \times 10^{-4}$ & $8.94 \times 10^{-5}$ & $7.39 \times 10^{-4}$ & $7.39 \times 10^{-3}$ \\
$\mathrm{R}^{2}$ & 0.999 & 0.999 & 0.994 & 0.991 \\
\hline
\end{tabular}

depende de la naturaleza o constitución del alimento (RIZVI, 1995). Se puede observar que en el caso de polvo de pulpa de lulo, su comportamiento no fue muy pronunciado talvez debido a las modificaciones físicas o químicas que ocurren durante el proceso de secado de pulpa en la forma de polvo, ya que se sabe que las isotermas son influenciadas por la composición, estructura física (cristalina o amorfa), y pretratamiento, como es el caso de un alimento procesado.

Gabas, Menegalli y Telis-Romero (2000) reportaron que la dependencia de las isotermas con la temperatura fue diferente para pulpa y cáscaras de ciruela. Sus resultados mostraron que las isotermas de pulpa de ciruela fueron levemente dependientes de la temperatura, mientras que las obtenidas para la cáscara de la ciruela fue claramente independiente de esta variable.

No fue observada intersección de las isotermas ni efecto del inverso de la temperatura para polvos de pulpa de lulo, pero hubo una tendencia al cruzamiento en altas actividades de agua. Algunos estudios han reportado este cruzamiento en función de la temperatura en actividades de agua por encima de 0.7 para productos con altos contenidos de azúcar, como es el caso de frutas (TELIS-ROMERO et al., 2005; TSAMI et al., 1990), los cuales son explicados por un aumento en la solubilidad de azúcares en agua causados por el aumento de temperatura.

También las isotermas obtenidas para polvos de pulpa de lulo que contienen maltodextrina o goma Arábiga producen isotermas sigmoidales del tipo III. Sin embargo, se encontraron diferencias considerables entre isotermas de polvo de lulo con aditivos (MD or GA) y las de pulpa pura. Este comportamiento es claramente observado en la Figura 2 que muestra el efecto de aditivos a $20^{\circ} \mathrm{C}$. La humedad de equilibrio de las muestras que contienen aditivos fue significativamente menor, que la de lulo puro para una determinada actividad de agua. Comportamiento similar fue observado por Gabas et al. (2007) y por Silva, Sobral y Kiechbush (2006), para pulpa de piña y camu-camu encapsulados con maltodextrina respectivamente.

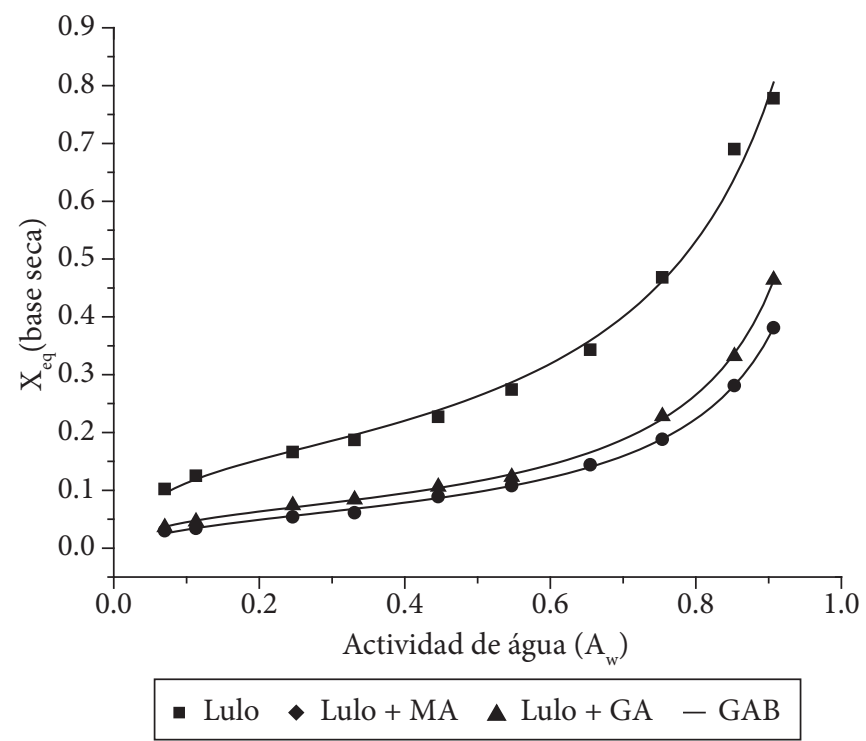

Figura 2. Influencia de adición de MD y GA sobre las isotermas de sorción de deshidratados de pulpa de lulo a $20^{\circ} \mathrm{C}$. 
Observando los resultados del ajuste del modelo de GAB, las isotermas para pulpa con aditivos presentan valores menores de humedad para la monocapa que la de pulpa pura.

Las muestras de polvo de pulpa de lulo deshidratado con adición de maltodextrina $(\mathrm{PL}+\mathrm{MD})$ presentaron valores de $\mathrm{X}_{\mathrm{m}}$ entre 4.9 y $7.4 \%$ (base seca), mientras que las muestras con goma arábiga $(\mathrm{PL}+\mathrm{GA})$ presentaron un rango de $\mathrm{X}_{\mathrm{m}}$ entre 5.5 y $7.8 \%$ (base seca). Los valores de monocapa para ambos materiales $(\mathrm{PL}+\mathrm{MD}$ and $\mathrm{PL}+\mathrm{GA})$ no presentan una clara dependencia con la temperatura.

Pérez-Alonso et al. (2006) obtuvieron valores de $\mathrm{X}_{\mathrm{m}}$ entre 6.96 y $7.35 \%$ (base seca) para maltodextrina pura (DE 10) y entre 8.11 a $11.00 \%$ (base seca) para goma arábiga, en el rango de temperatura entre 25 y $40^{\circ} \mathrm{C}$, valores que están de acuerdo con los obtenidos en este trabajo, dado que las muestras de PL + MD presentaron valores menores de $\mathrm{X}_{\mathrm{m}}$ que las muestras de $\mathrm{PL}+\mathrm{GA}$. Silva, Sobral y Kiechbush (2006) observaron que la adición de $30 \%$ de maltodextrina DE 20 en pulpa de camu-camu redujo $\mathrm{X}_{\mathrm{m}}$ de 15.8 al 6.5\% (base seca), un efecto muy similar fue observado con la pulpa de lulo. Righetto y Netto (2005) calcularon valores de agua de la monocapa entre 4.52 y $5.43 \%$ (base seca) para jugo de acerola seca por aspersión con $20 \%$ de maltodextrina DE 25 a temperaturas de 25,35 y $45^{\circ} \mathrm{C}$. Se observaron pequeñas diferencias de $\mathrm{X}_{\mathrm{m}}$ al emplear $20 \%$ de goma arábiga en lugar de maltodextrina: los valores del contenido de humedad de la monocapa variaron entre 4.80 y 5.44 (\% base seca), pero presentaron una tendencia a disminuir con el aumento de temperatura, lo cual se observó con la maltodextrina. Para el jugo de acerola encapsulado con goma arábiga se presentó una dependencia de $\mathrm{X}_{\mathrm{m}}$ con temperatura contraria al comportamiento reportado por Pérez-Alonso et al. (2006) para este mismo encapsulante.

Para muestras $\mathrm{PL}+\mathrm{MD}$, el parámetro $\mathrm{C}$ disminuye de 9.81 en $20^{\circ} \mathrm{C}$, a 3.11 en $50{ }^{\circ} \mathrm{C}$, mientras que para muestras $\mathrm{PL}+\mathrm{GA}$, los valores cambiaron de 15.15 en $20^{\circ} \mathrm{C}$, a 11.1 en $50{ }^{\circ} \mathrm{C}$. Pérez-Alonso et al. (2006) obtuvieron magnitudes similares, entre 5.26 y 18.83, para el valor de C con la adición de maltodextrina y goma Arábiga. Estos autores encontraron un comportamiento diferente del parámetro $\mathrm{C}$ en función de la temperatura: para GA, C se incrementa y para $\mathrm{MD}, \mathrm{C}$ disminuye con el aumento de la temperatura, lo cual puede ser atribuído al hecho de que el parámetro $\mathrm{C}$ no tenga un significado físico y que su variación con temperatura pueda ser resultado de una compensación matemática entre $\mathrm{C}$ y K. Righetto y Netto (2005) también observaron una variación irregular de C con la temperatura para muestras con maltodextrina. Por otro lado, muestras con goma Arábiga presentaron una disminución clara de $\mathrm{C}$ con el aumento de temperatura.

El parámetro K prácticamente no fue afectado por la presencia de aditivos, presentando valores entre 0.912 y 0.992 para muestras de PL + GA y entre 0.886 y 0.973 para muestras de PL + MD. Righetto y Netto (2005) no observaron variaciones de $\mathrm{K}$ relacionadas con la presencia de aditivos, mientras que Pérez-Alonso et al. (2006) encontraron en general, una ligera disminución de los valores de $\mathrm{K}$, siendo mayor para GA que para $\mathrm{MD}$, hecho que también se observó en los deshidratados de lulo con los mismos aditivos, evaluados en este trabajo.
La presencia de aditivos en pulpa de lulo probablemente modifica el balance de sitios hidrofílicos/hidrofóbicos, promoviendo una menor cantidad de agua adsorbida. Considerando la adición de biopolímeros, los procesos de sorción de agua también envuelven cambios estructurales de la matriz debido a hinchamiento (PÉREZ-ALONSO et al., 2006). Adhikari et al. (2004) encontraron que la maltodextrina altera la adherencia de la superficie de azúcares de bajo peso molecular y ácidos orgánicos, evitando que estos se fijen en las paredes de los equipos de secado, por lo que se le considera un aditivo efectivo en estos procesos. Basado en algunos parámetros, como higroscopicidad (Hygroscopicity), grado de aglomeración (degree of caking), grado de dispersión (dispersability), fluidez (flowability) y adherencia (stickiness), los cuales fueron definidos como parámetros de calidad de polvo de mango deshidratado al vacío, Jaya y Das (2004) recomiendan una composición optima de la mezcla de 0.43 a $0.57 \mathrm{~kg} \mathrm{MD} \mathrm{kg}^{-1}$ de polvo de mango.

Es importante notar que el equivalente de dextrosa en la maltodextrina ejerce una influencia directa sobre la temperatura de transición vítrea del sistema, valores menores de DE conducen a mayores valores de Tg. Este punto debe ser considerado cuando se comparan los resultados obtenidos en diferentes trabajos, ya que la MD ha sido utilizada como aditivo en el proceso de secado con maltodextrinas que presentan valores de DE entre 10 (PÉREZ-ALONSO et al., 2006) y 25 (RIGHETTO; NETTO, 2005).

La dependencia del calor isostérico de sorción de agua $\left(\mathrm{q}_{\mathrm{st}}\right)$ con el contenido de humedad para polvo de pulpa de lulo con y sin aditivos se muestra en la Figura 3.

En estas curvas se observa que la cantidad de calor requerida para remover agua del polvo de lulo va desde un contenido de humedad de $0.3 \mathrm{~kg} \cdot \mathrm{kg}^{-1}$ en base seca hasta $0.025 \mathrm{~kg} \cdot \mathrm{kg}^{-1}$. Para valores pequeños de humedad $\left(<0.1 \mathrm{~kg} \cdot \mathrm{kg}^{-1}\right.$ base seca), los valores de $\mathrm{q}_{\mathrm{st}}\left(\mathrm{kJ} \cdot \mathrm{mol}^{-1}\right)$ son altamente negativos, lo que significa que la sorción es una reacción espontánea. De acuerdo con Tsami (1991), los valores más negativos de $\mathrm{q}_{\mathrm{st}}$, indican un mayor

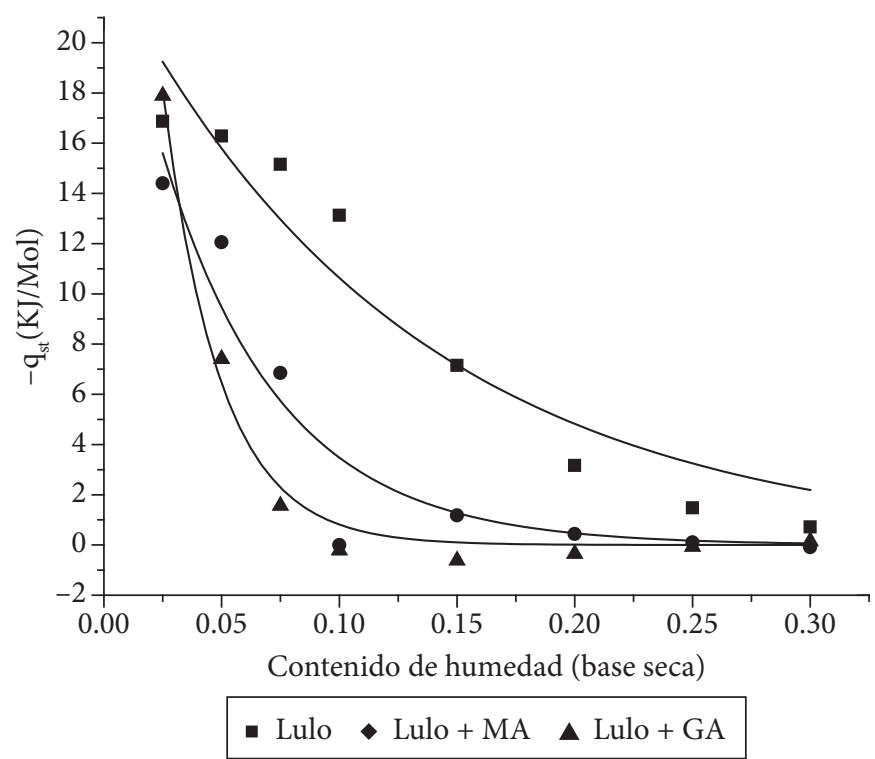

Figura 3. Influencia de encapsulantes en el calor isostérico de sorción de polvo de lulo. 
Tabla 5. Parámetros estimados por la ecuación de Tsami (Ecuación 5) para todas las muestras.

\begin{tabular}{lccc}
\hline Muestra & $\mathrm{q}_{0}\left(\mathrm{~kJ} \cdot \mathrm{mol}^{-1}\right)$ & $\mathrm{X}_{0}(\%$ base seca $)$ & $\mathrm{R}^{2}$ \\
\hline PL & 23.44 & 0.126 & 0.924 \\
PL + GA & 25.72 & 0.049 & 0.913 \\
PL + MD & 50.84 & 0.024 & 0.988 \\
\hline
\end{tabular}

grado de agua ligada en la superficie del alimento. En casi todo el rango de humedad, los valores de $\mathrm{q}_{\mathrm{st}}$ para muestras de PL son menores (más negativos) que los calculados para muestras de $\mathrm{PL}+\mathrm{GA}$ y PL + MD, lo que significa que el agua ligada para polvo de pulpa pura de lulo es mayor que en presencia de agentes encapsulantes. Conforme el contenido de humedad disminuye, las muestras que contienen aditivos presentan una disminución rápida de $\mathrm{q}_{\mathrm{st}}$, de forma que las curvas alcanzan prácticamente un valor similar altamente negativo para contenidos de humedad de $0.025 \mathrm{~kg} \cdot \mathrm{kg}^{-1}$ (base seca). Para humedades mayores de $0.1 \mathrm{~kg} \cdot \mathrm{kg}^{-1}$ (base seca), $\mathrm{q}_{\mathrm{st}}$ presentó valores positivos para pulpa de lulo con materiales encapsulantes, principalmente para $\mathrm{PL}+\mathrm{MD}$. Es importante notar que, para contenidos de humedad menores de $0.2 \mathrm{~kg} \mathrm{~kg}^{-1}$ (base seca), el aumento de la negatividad en el calor isostérico de sorción debe ser tenido en cuanta para la simulación de la energía requerida en el proceso de secado, lo que de manera general, son menores para los polvos con agentes encapsulantes. Se supone que para contenidos de humedad por encima de $0.3 \mathrm{~kg} \cdot \mathrm{kg}^{-1}$ (base seca) las diferencias entre $\mathrm{q}_{\mathrm{st}}$ para todas las muestras tienden a desaparecer. El comportamiento presentado en la Figura 3 puede ser explicado en términos de un efecto plastificante de las moléculas de agua. El trabajo de Telis y Sobral (2001) mostró que un aumento en el contenido de humedad de cero a $30 \%$ (base seca) provocó una importante reducción en la temperatura de transición vítrea de pulpa de piña liofilizada de 63 a $-70{ }^{\circ} \mathrm{C}$. De otro lado, la adición de compuestos de alto peso molecular como MD o GA deberá minimizar efectivamente el elevado efecto plastificante.

Los valores experimentales de $q_{s t}$ se ajustaron a la Ecuación 5, a partir de la cual se obtuvieron las constantes $\mathrm{q}_{0} \mathrm{yX}_{0}$, La Tabla 5 presenta estos valores, así como el coeficiente de correlación $\left(\mathrm{R}^{2}\right)$.

De manera general, los valores del coeficiente de determinación $\left(\mathrm{R}^{2}\right)$ fueron satisfactorios. Los valores calculados de $\mathrm{q}_{0}$ fueron mayores para $\mathrm{PL}+\mathrm{GA}$ y $\mathrm{PL}+\mathrm{MD}$ que para $\mathrm{PL}$, probablemente debido a las fuertes interacciones de los componentes del alimento con la primera molécula de agua de la monocapa en este tipo de productos. Estos resultados fueron aproximadamente dos veces mayores en comparación con los obtenidos para frutas como pulpa de uva (GABAS; TELIS-ROMERO; MENEGALLI, 1999), ciruelas (GABAS; MENEGALLI; TELIS-ROMERO, 2000) y vegetales (KIRANOUDIS et al., 1993).

\section{Conclusiones}

El modelo de GAB para isotermas fue adecuado para describir datos experimentales para polvos de pulpa de lulo con y sin aditivos (PL; PL + GA; PL + MD). En la misma actividad de agua, muestras PL + GA y PL + MD presentaron menor contenido de humedad de equilibrio y fueron menos afectadas por el cambio de temperatura. El calor isostérico de sorción de polvos de pulpa con aditivos fue mayor (menos negativo) que para los polvos de pulpa pura de lulo, suponiendo que hay más sitios polares activos en el producto sin la adicción de GA o MD. Una relación exponencial empírica puede describir la dependencia que el calor de sorción tiene en función del contenido de humedad.

\section{Agradecimientos}

Los autores agradecen al CNPq (Procesos No 474626/ 2004-0, No 491504/2004-7 y No 471223/2006-9) por el soporte financiero.

\section{Referencias}

ADHIKARI, B. et al. Effect of addition of maltodextrin on drying kinetics and stickiness of sugar and acid-rich foods during convective drying experiments and modelling. Journal of Food Engineering, v. 62, p. 53-68, 2004.

ASSOCIATION OF OFFICIAL AGRICULTURAL CHEMISTS AOAC. Official Methods of Analysis. $15^{\text {th }}$ ed. Washington, D.C., 1990.

BEMILLER, J. N.; WHISTLER, R. L. Carbohydrates. In: FENEMMA, O. R. (Ed.) Food Chemistry. $3^{\text {rd }}$ ed. New York: Marcel Dekker, 1996. p.157-224.

BHANDARI, B. R. et al. Spray drying of concentrated fruit juices. Drying Technology, v. 11, p. 1081-1092, 1993.

DIOSADY, L. L. Moisture sorption isotherms of canola meals, and applications to packaging. Journal of Food Science, v. 61, p. 204-208, 1996.

GABAS, A. L.; MENEGALLI, F. C.; TELIS-ROMERO, J. Water sorption enthalpy-entropy compensation based on isotherms of plum skin and pulp. Journal of Food Science, v. 65, p. 680-684, 2000.

GABAS, A. L. et al. Effect of maltodextrin and arabic gum in water vapor sorption thermodynamic properties of vacuum dried pineapple pulp powder. Journal of Food Engineering, v. 82, p. 246-252, 2007.

GABAS, A. L.; TELIS-ROMERO, J.; MENEGALLI, F. C. Thermodynamic models for water sorption by grape skin and pulp. Drying Technology, v. 17, p. 961-974, 1999.

GOMEZ, C. R. et al. Caracterización y normalización del lulo de castilla. In: SEMINARIO NACIONAL E INTERNACIONAL DE FRUTALES, 5., 2004, Manizales, CO. Anais... Manizales, CO: Universidad Nacional de Colombia - UNAL, 2004. p. 161-168.

JAYA, S.; DAS, H. Effect of maltodextrin, glycerol monostearate and tricalcium phosphate on vacuum dried mango powder properties. Journal of Food Engineering, v. 63, p. 125-134, 2004.

JOWITT, R. Physical properties of foods. New York: Applied Science Publishers, 1983. 425p.

KIRANOUDIS, C. T. et al. Equilibrium moisture content and heat of desorption of some vegetables. Journal of Food Engineering, v. 20, p. 55-74, 1993.

LEWICKI, P. The applicability of the GAB model to food water sorption isotherms. International Journal of Food Science and Technology, v. 32, p. 553-557, 1997.

LOMAURO, C. J.; BAKSHI, A. S.; LABUZA, T. P. Evaluation of food moisture isotherm equations. Part I: fruit, vegetable and meat 
products. Lebensmittel-Wissenschaft Und. Technologie, v. 18, p. 111-117, 1985.

LOPES-FILHO, J. F. et al. Sorption Isotherms of Alligator's Meat (Caiman crocodrilus yacare). Journal of Food Engineering, v. 19, p. 119-126, 2002.

MCLAUGHLIN, C. P.; MAGEE, T. R. A. The determination of sorption isotherm and the isosteric heats of sorption for potatoes. Journal of Food Engineering, v. 35, p. 267-280, 1998.

MOREIRA, R. et al. Thermodynamic analysis of experimental sorption isotherms of loquat and quince fruits. Journal of Food Engineering, v. 88, p. 514-521, 2008.

NICOLETI, J. F.; TELIS-ROMERO, J.; TELIS, V. R. N. Air-drying of fresh and osmotically pre-treated pineapple slices: fixed air temperature versus fixed slice temperature drying kinetics. Drying Technology, v. 19, p. 2175-2191, 2001.

PÉREZ-ALONSO, C. et al. Thermodynamic analysis of the sorption isotherms of pure and blended carbohydrate polymers. Journal of Food Engineering, v. 77, p. 753-760, 2006.

REINECCIUS, G. A. Carbohydrates for flavor encapsulation. Food Technology, v. 46, p. 144-152, 1991.

RIGHETTO, A. M.; NETTO, F. M. Effect of encapsulating materials on water sorption, glass, transition and stability of juice from immature acerola. International Journal of Food Properties, v. 8, p. 337-346, 2005.

RIZVI, S. S. H. Thermodynamic properties of foods in dehydration. In: RAO, M. A.; RIZVI, S. S. H. (Ed.). Engineering properties of foods. New York: Academic Press, 1995. Chap 4.
ROCKLAND, L.; BEUCHAT, L. Water activity: theory and applications to food. New York: Marcel Decker, 1987.

SILVA, M. A.; SOBRAL, P. J. A.; KIECKBUSCH, T.G. State diagrams of freeze-dried camu-camu (myrciaria dubia (hbk) mc vaugh) pulp with and without maltodextrin addition. Journal of Food Engineering, v. 77, p. 426-432, 2006.

SOBRAL, P. J. A.; LEBERT, A.; BIMBENET, J. J. Isothermes de désorption de la pomme de terre entre 40 et $70{ }^{\circ} \mathrm{C}$. Science des Aliments, v. 19, p. 711-720, 1999.

STATSOFT. Statistica for Windows: computer program manual. Tulsa, OK, 1995.

TAMAYO, A. et al. Frutales de Clima Frio Moderado. Colombia: Corpoica, 2001.

TELIS, V. R. N. et al. Water sorption thermodynamic properties applied to persimmon skin and pulp. Thermochimica Acta, v. 343, p. 49-56, 2000.

TELIS, V. R. N.; SOBRAL, P. J. A. Glass transitions and state diagram for freeze-dried pineapple. Lebensmittel-Wissenschaft undTechnologie, v. 34, p. 199-205, 2001.

TELIS-ROMERO, J. et al. Enthalpy-entropy compensation based on isotherms of mango. Ciência e Tecnologia de Alimentos, v. 25, p. 293-303, 2005.

TSAMI, E. Net isosteric heat of sorption in dried fruits. Journal of Food Engineering, v. 14, p. 327-335, 1991.

TSAMI, E. et al. Heat of sorption of water in dried fruits. International. Journal of Food Science and Technology, v. 25, p. 350-359, 1990. 